\title{
DIFFERENTIAL INEQUALITIES AND CARATHÉODORY FUNCTIONS
}

\author{
BY SANFORD MILLER ${ }^{1}$
}

Communicated by Robert Bartle, April 15, 1974

\begin{abstract}
The author proves a very general result from which it is possible to show that a regular function satisfying a differential inequality of a certain type is necessarily a Carathéodory function. This result has applications in the theory of univalent functions.
\end{abstract}

Let $\mathscr{P}$ denote the class of Carathéodory functions; that is, functions $p(z)=1+p_{1} z+p_{2} z^{2}+\cdots$ regular in the unit disc $\Delta$, and for which $\operatorname{Re} p(z)>0$.

In a recent paper [2] it was shown that if $p(z)=1+p_{1} z+p_{2} z^{2}+\cdots$ is regular in $\Delta$, with $p(z) \neq 0$ in $\Delta$, and if $\alpha$ is a real number, then for $z \in \Delta$

$$
\operatorname{Re}\left[p(z)+\alpha\left(z p^{\prime}(z) \mid p(z)\right)\right]>0 \Rightarrow \operatorname{Re} p(z)>0 ;
$$

that is, $p(z) \in \mathscr{P}$.

In this note we replace the differential inequality in (1) by a much more general condition which will still imply that $p(z)$ is a Carathéodory function.

Definition 1. Let $u=u_{1}+u_{2} i$ and $v=v_{1}+v_{2} i$, and let $\Psi$ be the set of functions $\psi(u, v)$ satisfying:

(a) $\psi(u, v)$ is continuous in a domain $D$ of $C \times C$;

(b) $(1,0) \in D$ and $\operatorname{Re} \psi(1,0)>0$;

(c) $\operatorname{Re} \psi\left(u_{2} i, v_{1}\right) \leqq 0$ when $\left(u_{2} i, v_{1}\right) \in D$ and $v_{1} \leqq-1 / 2\left(1+u_{2}^{2}\right)$.

We denote by $\Phi$ the subset of $\Psi$ which satisfies (a), (b) and the following condition:

$\left(\mathrm{c}^{\prime}\right) \operatorname{Re} \psi\left(u_{2} i, v_{1}\right) \leqq 0$ when $\left(u_{2} i, v_{1}\right) \in D$ and $v_{1} \leqq 0$.

EXAMPLES. It is easy to check that each of the following functions are in $\Psi$.

$$
\begin{aligned}
& \psi_{1}(u, v)=u+\alpha v / u, \alpha \text { real, with } D=[C-\{0\}] \times C . \\
& \psi_{2}(u, v)=u^{2}+v \text { with } D=C \times C . \\
& \psi_{3}(u, v)=u+\alpha v, \alpha \geqq 0, \text { with } D=C \times C . \\
& \psi_{4}(u, v)=u-v / u^{2} \text { with } D=[C-\{0\}] \times C . \\
& \psi_{5}(u, v)=-\ln \left(\frac{1}{2}-v\right) \text { with } D=C \times\left\{\left(v_{1}, v_{2}\right) \mid v_{1}<\frac{1}{2}\right\} .
\end{aligned}
$$

AMS (MOS) subject classifications (1970). Primary 30A04, 30A20, 34A40; Secondary $30 \mathrm{~A} 32$.

Key words and phrases. Carathéodory functions, univalent functions.

1 The author acknowledges support received from the National Academy of Sciences through its exchange program with the Academy of the Socialist Republic of Romania. 
Note that $\psi_{1}, \psi_{2}, \psi_{3}$ and $\psi_{4}$ are also in $\Phi$ but $\psi_{5} \notin \Phi$. The set $\Phi$ is thus a proper subset of $\Psi$. Though some generality is lost in considering the class $\Phi$ as opposed to considering $\Psi$, the former is much easier to work with algebraically.

DEFINITION 2. Let $p(z)=1+p_{1} z+p_{2} z^{2}+\cdots$ be regular in $\Delta$ and let $\psi \in \Psi$ with corresponding domain $D$. We denote by $\mathscr{P}(\psi)$ those functions $p(z)$ that satisfy:

(i) $\left(p(z), z p^{\prime}(z)\right) \in D$, and

(ii) $\operatorname{Re} \psi\left(p(z), z p^{\prime}(z)\right)>0$,

when $z \in \Delta$.

Note that $\mathscr{P}(\psi)$ is not empty, since for all $\psi \in \Psi$ it is true that $p(z)=1+$ $p_{1} z \in \mathscr{P}(\psi)$ for $p_{1}$ sufficiently small (depending on $\psi$ ). It appears further that most $\psi \in \Psi$ provide a large number of other functions in $\mathscr{P}(\psi)$.

Our main result is the following theorem.

THEOREM 1. For any $\psi \in \Psi, \mathscr{P}(\psi) \subset \mathscr{P}$.

In other words the Theorem states that if $\psi \in \Psi$, with corresponding domain $D$, and if $\left(p, z p^{\prime}\right) \in D$ then

$$
\operatorname{Re} \psi\left(p(z), z p^{\prime}(z)\right)>0 \Rightarrow \operatorname{Re} p(z)>0 .
$$

Since $\Phi \subset \Psi$, we immediately have the following Corollary.

COROLlary. For any $\psi \in \Phi, \mathscr{P}(\psi) \subset \mathscr{P}$.

The proof of the Theorem is involved and will not be presented here. However an independent proof of the Corollary follows.

Let $p(z) \in \mathscr{P}(\psi)$, and assume there exists a point $z_{0}=r_{0} \exp \left(i \theta_{0}\right) \in \Delta$ such that $\operatorname{Re} p(z) \geqq 0$ for $|z| \leqq r_{0}$, and $\operatorname{Re} p\left(z_{0}\right)=0$. Thus $p\left(z_{0}\right)=a i$, where $a$ is a real number. We now show that $z_{0} p^{\prime}\left(z_{0}\right)=k$, where $k \leqq 0$. Since the result is true if $p^{\prime}\left(z_{0}\right)=0$, we need only consider the case $\overline{p^{\prime}}\left(z_{0}\right) \neq 0$. The curve $p\left(r_{0} e^{i \theta}\right)$ is tangent to the imaginary axis at $z_{0}$, and so we have $\arg z_{0} p^{\prime}\left(z_{0}\right)=\pi$; that is $z_{0} p^{\prime}\left(z_{0}\right)=k$, where $k<0$. Hence at $z_{0}$ we have $\operatorname{Re} \psi\left(p, z p^{\prime}\right)=\operatorname{Re} \psi(a i, k)$ with $a$ real and $k \leqq 0$. But this implies that $\operatorname{Re} \psi\left(p, z p^{\prime}\right) \leqq 0$ at $z=z_{0}$, which is a contradiction of the fact that $p(z) \in \mathscr{P}(\psi)$. Hence $\operatorname{Re} p(z)>0$ for $z \in \Delta$.

REMARKS. If we apply the Theorem (or the Corollary) to the example $\psi_{1}(u, v)$, we obtain condition (1). Applying it to $\psi_{2}, \psi_{3}$ and $\psi_{4}$ we obtain respectively:

$$
\operatorname{Re}\left[p^{2}(z)+z p^{\prime}(z)\right]>0 \Rightarrow \operatorname{Re} p(z)>0 ;
$$

$$
\operatorname{Re}\left[p(z)+\alpha z p^{\prime}(z)\right]>0 \text {, with } \alpha \geqq 0 \Rightarrow \operatorname{Re} p(z)>0 \text {, }
$$

and

$$
p(z) \neq 0 \text { and } \operatorname{Re}\left[p(z)-z p^{\prime}(z) / p^{2}(z)\right]>0 \Rightarrow \operatorname{Re} p(z)>0 .
$$


We see that for different $\psi \in \Psi$ we can obtain different differential conditions for $p(z)$ to be a Carathéodory function. By appropriately choosing $\psi \in \Psi$ we can define many new subclasses of $\mathscr{P}$ and can prove many properties of the class $\mathscr{P}$.

The theorem has many applications in the theory of univalent functions. If we set $p(z)=z f^{\prime}(z) / f(z)$ in Theorem 1 , we see from (2) that each $\psi \in \Psi$ generates a subclass of starlike functions. In particular $\psi_{1}(u, v)=u+$ $\alpha v / u$ generates the class of alpha-convex functions [2]. Similarly by setting $p(z)=e^{i y} z f^{\prime}(z) \mid f(z)$, where $|\gamma|<\frac{1}{2}$, or $p(z)=f^{\prime}(z) / g^{\prime}(z)$, where $g(z)$ is convex, and using slightly modified forms of Definitions 1 and 2 and Theorem 1, we can generate many new subclasses of spiral-like and close-to-convex functions, respectively. These results, the proof of Theorem 1 , and other applications will appear in a forthcoming paper [1].

\section{REFERENCES}

1. Z. Lewandowski, S. Miller and E. Zlotkiewicz, Generating functions for classes of univalent functions, (to appear).

2. S. S. Miller, P. Mocanu and M. O. Reade, All alpha-convex functions are starlike, Rev. Roumaine Math. Pures Appl. 17 (1972), 1395-1397.

Department of Mathematics, State University of New York, Brockport, NEW YORK 14420 